\title{
Mitochondrial dysfunction suppresses p53 expression via calcium-mediated nuclear factor-kB signaling in HCT116 human colorectal carcinoma cells
}

\author{
Young-Kyoung Lee ${ }^{\#}$ Eui-Yeun Yi $i^{\#}$ Shi-Young Park, Won-Jun Jang, Yu-Seon Han, Myeong-Eun Jegal E Yung-Jin Kim * \\ Department of Molecular Biology, College of Natural Sciences, Pusan National University, Busan 46241, Korea
}

\begin{abstract}
Mitochondrial DNA (mtDNA) mutations are often observed in various cancer types. Although the correlation between mitochondrial dysfunction and cancer malignancy has been demonstrated by several studies, further research is required to elucidate the molecular mechanisms underlying accelerated tumor development and progression due to mitochondrial mutations. We generated an mtDNA-depleted cell line, $\rho^{0}$, via long-term ethidium bromide treatment to define the molecular mechanisms of tumor malignancy induced by mitochondrial dysfunction. Mitochondrial dysfunction in $\rho^{0}$ cells reduced drug-induced cell death and decreased the expression of pro-apoptotic proteins including p53. The p53 expression was reduced by activation of nuclear factor- $\kappa \mathrm{B}$ that depended on elevated levels of free calcium in $\mathrm{HCT} 116 / \rho^{0}$ cells. Overall, these data provide a novel mechanism for tumor development and drug resistance due to mitochondrial dysfunction. [BMB Reports 2018; 51(6): 296-301]
\end{abstract}

\section{INTRODUCTION}

Mitochondria are the main energy-producing organelles, which regulate cellular metabolism, cell death, and production of reactive oxygen species (ROS) (1-3). Mitochondria contain their own DNA (called mitochondrial DNA [mtDNA]) carrying 13 enzyme-encoding genes including those related to

*Corresponding author. Tel: +82-51-510-2176; Fax: +82-51-5139258; E-mail: yjinkim@pusan.ac.kr

"These authors contributed equally to this work.

Current address: Young-Kyoung Lee, Department of Biochemistry and Molecular Biology, Ajou University School of Medicine, Suwon 16499, Korea; Shi-Young Park, Korea Mouse Metaboilc Phenotyping Center, Lee Gil Ya Cancer and Diabetes Institute, Gachon University, Incheon 21999, Korea; Won-Jun Jang, College of Pharmacy, Keimyung University, Daegu 42601, Korea

https://doi.org/10.5483/BMBRep.2018.51.6.232

Received 7 December 2017, Revised 2 January 2018, Accepted 5 March 2018

Keywords: Calcium, Mitochondrial dysfunction, NF-кB, p53, $\rho^{0}$ cell oxidative phosphorylation, transfer RNAs, and ribosomal RNAs (4). As mitochondria are the major ROS production sites, mtDNA is often directly exposed to highly reactive ROS (5). Despite the presence of antioxidant systems such as superoxide dismutase, glutathione, and peroxiredoxin in mitochondria, mtDNA is prone to damage by ROS (5). Consequently, numerous mtDNA mutations have been reported in various cancer types (6). These mutations are linked to both primary tumor growth and metastasis (7). However, additional research is required to elucidate the molecular mechanisms of tumor growth and progression induced by mitochondrial mutations.

The tumor suppressor p53 is a transcription factor, which plays a decisive role in cell cycle regulation and apoptosis (8, 9). The importance of p53 in tumorigenesis is reinforced by reports suggesting that p53 is highly mutated in over $50 \%$ of cancer cells. Mutations in p53 result not only in the loss of normal protein function, but also lead to carcinogenesis (10). Recently, several researchers reported that p53 expression is tightly linked to mitochondrial homeostasis and function (11-13), suggesting that p53 regulates mitochondrial membrane potential, and loss of p53 results in a significant increase in mtDNA mutations. Furthermore, the relationship between the loss of p53 and mitochondrial dysfunction has been reported in a variety of cancers (14-16).

Several recent reports have suggested possible molecular mechanisms underlying tumorigenesis caused by mitochondrial dysfunction. However, the mechanisms are still poorly elucidated. In the present study, we observed a reduced p53 expression in the mtDNA-depleted cellular model, HCT116 $/ \rho^{0}$, derived from the colorectal carcinoma cell line HCT116. This observation supports the theory that mitochondrial dysfunction leads to tumor progression and drug resistance via reduction of p53 expression.

\section{RESULTS}

Mitochondrial DNA-depleted HCT116/ $\rho^{0}$ cells are resistant to anticancer drug-induced apoptosis

Previously, several reports revealed that various cancer cells exhibited defective mitochondrial function $(5,6)$. In addition,

ISSN: 1976-670X (electronic edition)

Copyright (C) 2018 by the The Korean Society for Biochemistry and Molecular Biology

(c) This is an open-access article distributed under the terms of the Creative Commons Attribution Non-Commercial License (http://creativecommons.org/licenses/by-nc/4.0) which permits unrestricted non-commercial use, distribution, and reproduction in any medium, provided the original work is properly cited. 
cancer cells with mitochondrial dysfunction are more malignant than cancer cells with normal mitochondrial function (7). To investigate the molecular mechanism of drug resistance, one of the malignant phenotypes induced by mitochondrial dysfunction, we established the mtDNA-depleted cell line, $\rho^{0}$. The $\rho^{0}$ cell line is invaluable for the study of diseases related to mitochondrial dysfunction $(17,18)$. Mitochondrial DNAdepleted cells were established by treatment with $1 \mu \mathrm{g} / \mathrm{ml} \mathrm{EtBr}$ for 50 days. As shown in Fig. $1 \mathrm{~A}$, the number of mtDNA transcripts in $\mathrm{HCT} 116 / \rho^{0}$ cells was diminished by $\mathrm{EtBr}$ treatment. To examine the drug-resistant phenotype of HCT $116 / \rho^{0}$ cells, we tested whether HCT $116 / \rho^{0}$ cells were resistant to drug-induced cell death. Both parental HCT116 and $\mathrm{HCT} 116 / \rho^{\circ}$ cells were incubated with media containing taxol or doxorubicin for 3 days. The HCT116/ $\rho^{0}$ cells were more resistant to both taxol and doxorubicin than the parental cells (Fig. 1B and C). Furthermore, the expression of apoptotic proteins such as p53, Bax, and Bid was reduced, and the expression of the anti-apoptotic protein $\mathrm{Bcl} 2$ was up-regulated in HCT116/ $\rho^{0}$ cells (Fig. 1D). These results indicate that mtDNA-depleted HCT116/ $\rho^{0}$ cells exhibited a more drugresistant phenotype than their parental cells.
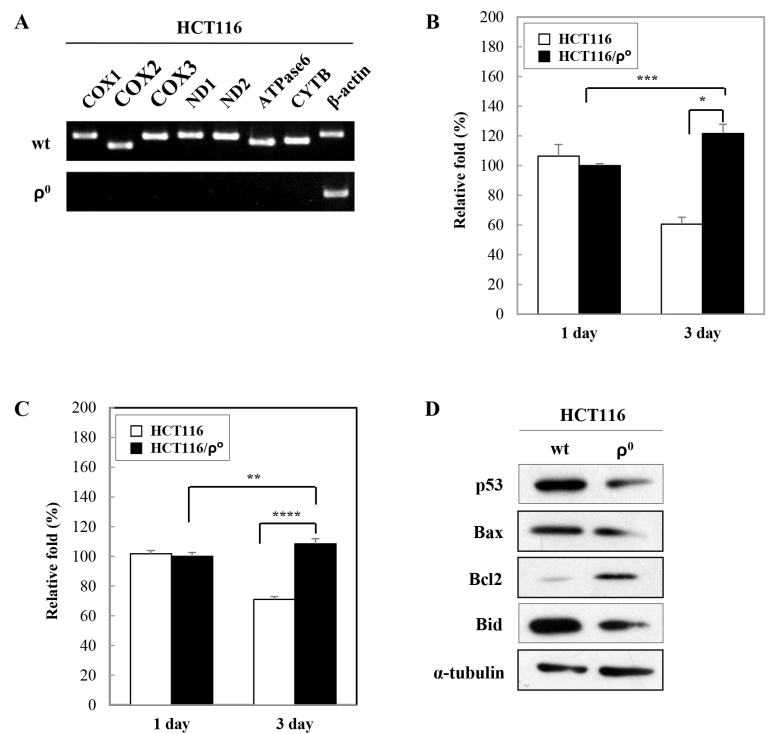

Fig. 1. Resistance to anticancer drug-induced apoptosis in HCT116/ $/ \rho^{0}$ cells. HCT116 cells were treated with ethidium bromide to generate HCT116/ $\rho^{\circ}$ cells. (A) Mitochondrial gene expression in HCT116/ $\rho^{0}$ cells. The viabilities of HCT116 and $\mathrm{HCT} 116 / \rho^{0}$ cells treated with taxol $(10 \mathrm{nM}, \mathrm{B})$ and doxorubicin $(1$ $\mu \mathrm{M}, \mathrm{C})$ for 3 ds. Error bars represent mean $\pm \mathrm{SD}$. $* \mathrm{P}<0.05$, ${ }^{* * P}<0.01,{ }^{* * * P}<0.001$ by Student's test. (D) Protein expression levels of pro-apoptotic and anti-apoptotic genes: COX1, cytochrome c oxidase 1; COX2, cytochrome c oxidase 2; COX3, cytochrome c oxidase 3; ND1, NADH dehydrogenase 1; ND2, $\mathrm{NADH}$ dehydrogenase 2; CYTB, cytochrome $\mathrm{b}$ oxidase.

\section{Reduction of p53 expression follows mitochondrial DNA depletion in HCT116/ $/ \rho^{0}$ cells}

Many studies show that loss of p53 function plays a crucial role in tumor malignancy $(19,20)$. As shown in Fig. 1 , the p53 expression was reduced in $\mathrm{HCT} 116 / \rho^{0}$ cells. This reduction in p53 levels was also observed at the mRNA level (Fig. 2A), suggesting that mtDNA depletion affects transcription of the p53 gene. It has been reported that p53 regulates mtDNA copy number and mitochondrial homeostasis $(11,12)$. Thus, we tested mtDNA depletion and p53 expression to verify that loss of p53 leads to mtDNA depletion during establishment of HCT116/ $\rho^{0}$ cells. If loss of p53 leads to mtDNA depletion as expected, p53 expression is reduced prior to mtDNA depletion in HCT116/ $\rho^{0}$ cells. Following the treatment of HCT116 cells with EtBr, p53 expression and mtDNA depletion were monitored by RT-PCR in a time-dependent manner. Unexpectedly, mtDNA depletion started after 4 days of EtBr treatment, while p53 expression was reduced after 18 days of $\mathrm{EtBr}$ treatment (Fig. 2B and C). This result indicates that mtDNA depletion affects p53 expression.

Reduction of p53 expression is mediated by elevated intracellular calcium level, and not elevated NADH levels Mitochondrial dysfunction leads to accumulation of
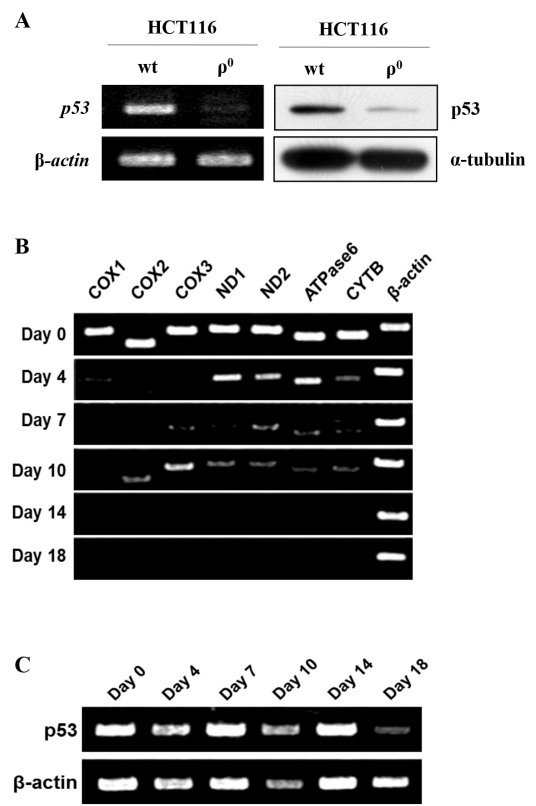

Fig. 2. Reduction in p53 expression following mitochondrial DNA depletion. (A) The mRNA and protein levels of p53 in HCT116 and $\mathrm{HCT} 116 / \rho^{0}$ cells. Time-dependent depletion of mitochondrial gene expression (B) and p53 expression (C). COX1, cytochrome $\mathrm{C}$ oxidase 1; COX2, cytochrome $\mathrm{c}$ oxidase 2; COX3, cytochrome $\mathrm{C}$ oxidase 3; ND1, NADH dehydrogenase 1; ND2, NADH dehydrogenase 2; CYTB, cytochrome b oxidase. 
intracellular $\mathrm{NADH}$ and calcium, which are both important intracellular signals $(21,22)$. To investigate whether these factors affected the decrease in p53 expression in the HCT $116 / \rho^{0}$ cells, intracellular NADH and calcium levels were measured. The intracellular NADH level was elevated in HCT116/ $\rho^{0}$ cells (Fig. 3A). If increased intracellular NADH level reduced p53 expression, it would have been reduced in $\mathrm{NADH}$-treated parental cells. However, NADH treatment did not reduce the p53 level in parental cells (Fig. 3C). Next, we measured the intracellular calcium levels in $\mathrm{HCT} 116 / \rho^{0}$ cells using a free calcium-binding fluorescent dye. As previously reported $(21,22)$, intracellular calcium levels in HCT116/ $\rho^{0}$ cells were higher than in parental cells (Fig. 3B). In order to test the effect of elevated intracellular calcium levels on p53 expression, we treated parental cells and HCT116/ $/ \rho^{\circ}$ cells with A23187 (a calcium ionophore) or BAPTA (a calcium chelator). In A23187-treated parental cells, the p53 level was lower than in untreated cells. The p53 level was restored by chelating the free intracellular calcium in $\mathrm{HCT} 116 / \rho^{0}$ cells (Fig. 3D). These results indicate that the reduction of p53 expression might be mediated by elevated calcium and not by NADH.
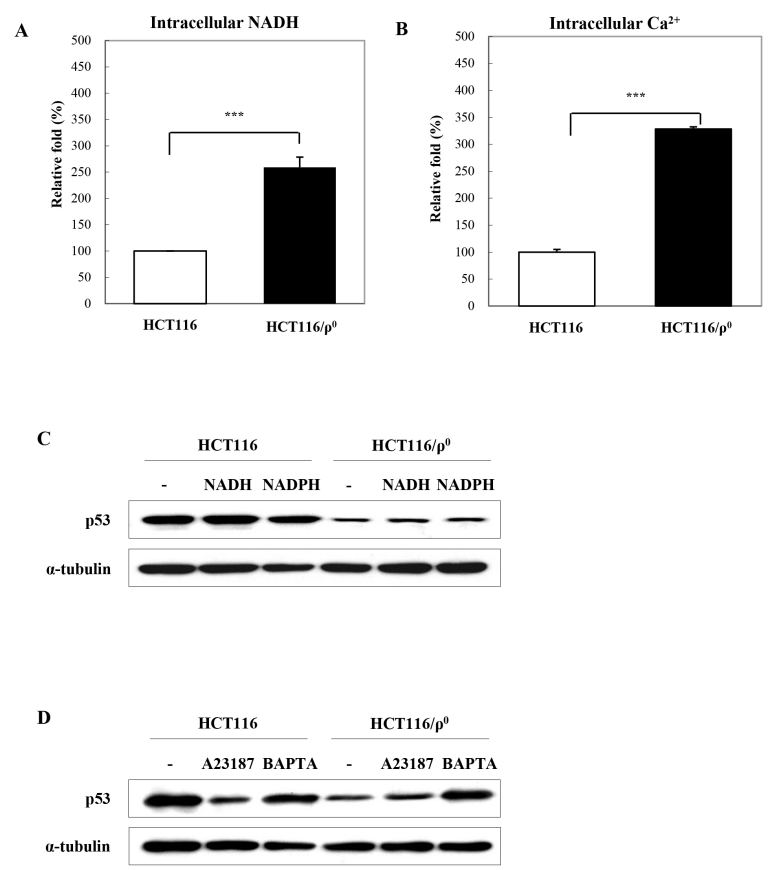

Fig. 3. Elevated intracellular calcium-mediated p53 reduction in HCT116/ $\rho^{0}$ cells. Cellular levels of NADH (A) and calcium (B). Error bars represent mean $\pm \mathrm{SD} . * \mathrm{P}<0.05, * * \mathrm{P}<0.01, * * * \mathrm{P}$ $<0.001$ by Student's test. (C) Protein expression of p53 in HCT116 and HCT116/ $\rho^{0}$ cells treated with NADH (1 mM) or NADPH $(1 \mathrm{mM})$. (D) Protein level of p53 in HCT116 and HCT116 $/ \rho^{0}$ cells treated with calcium ionophore, A23187 (5 $\left.\mu \mathrm{M}\right)$ or calcium chelator, BAPTA $(2 \mu \mathrm{M})$.
Activation of nuclear factor- $\kappa \mathrm{B}$ (NF- $\mathrm{\kappa} B$ ) by elevated cytosolic calcium reduces p53 expression in HCT116/ $\rho^{0}$ cells

According to previous reports, calcium-dependent calcineurin activation activated NF-KB (21), which reduced p53 levels (23, 24). Therefore, we assumed that elevated intracellular calcium level reduces p53 levels by activating NF- $\mathrm{KB}$. To confirm this assumption, we first examined NF- $\mathrm{KB}$ levels in both parental and $\mathrm{HCT} 116 / \rho^{0}$ cells. Both total and activated NF- $\mathrm{\kappa B}$ levels in HCT116/ $\rho^{0}$ cells were higher than in parental cells (Fig. 4A). Pyrrolidine dithiocarbamate, an NF-KB inhibitor (25), restored both protein and mRNA levels of p53 in HCT116/ $\rho^{0}$ cells (Fig. $4 \mathrm{~A}$ and $\mathrm{B}$ ). Next, we examined p53 and NF-KB levels after treatment with BAPTA to investigate the possibility that elevated calcium level reduces p53 expression through NF-KB. Chelation of elevated calcium in HCT $116 / \rho^{0}$ cells by BAPTA led to inhibition of NF- $\mathrm{KB}$ signaling (restoration of IKB protein levels and decrease in phosphorylated NF-אB levels; Fig. 4C),
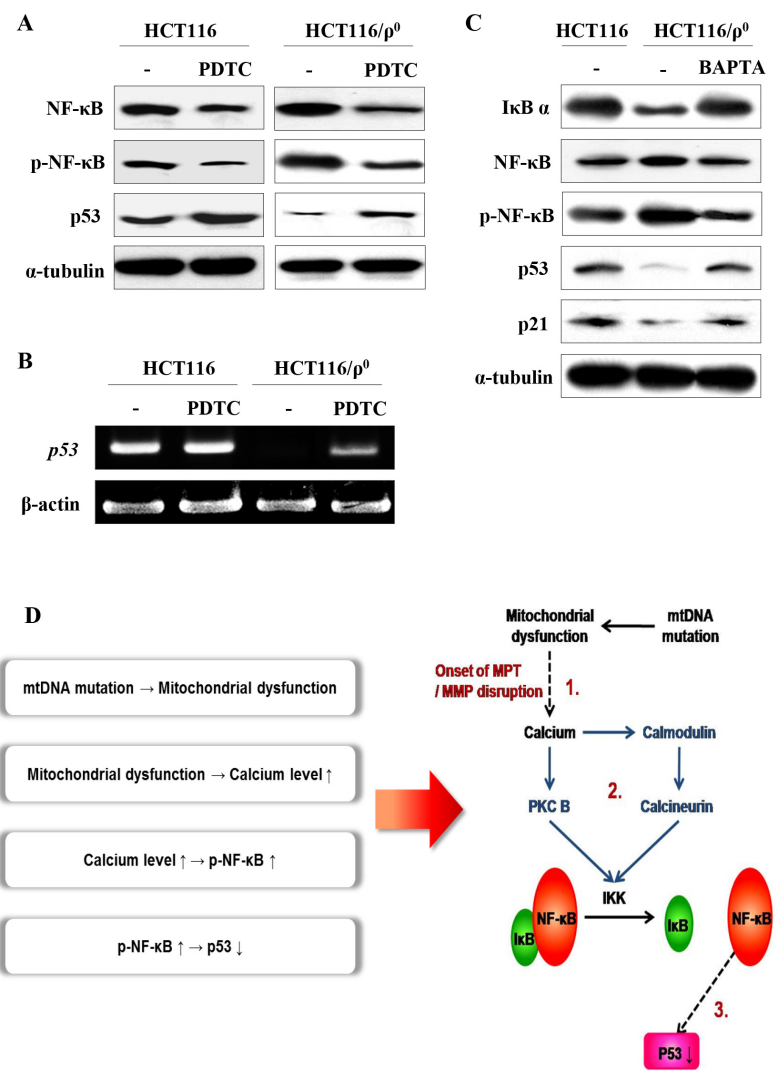

Fig. 4. Calcium-induced NF-kB-mediated p53 reduction in HCT116/ $\rho^{0}$ cells. (A) Protein levels of NF-KB and p53 in HCT116 and $\mathrm{HCT} 116 / \rho^{\circ}$ cells treated with pyrrolidine dithiocarbamate (PDTC, $2 \mu \mathrm{M}$ ). (B) mRNA level of p53 in HCT116 and HCT116/ $/ \rho^{0}$ cells treated with PDTC. (C) Protein levels of NF-KB, IKB, p53 and p21 in $\mathrm{HCT} 116 / \rho^{\circ}$ cells treated with PDTC. (D) Hypothetical working model for p53 reduction induced by mitochondrial dysfunction. 
which also rescued the expression of p53 and p21 in $\mathrm{HCT} 116 / \rho^{0}$ cells. These data indicate that activation of NF- $\kappa B$ by elevated intracellular calcium level reduced the p53 expression in HCT116/ $\rho^{0}$ cells.

\section{DISCUSSION}

Mitochondrial DNA mutations and mitochondrial dysfunction have been frequently described in various human cancers including renal, head and neck, breast, ovarian, liver, prostate, bladder, and colon cancers $(5,6)$. Mitochondrial dysfunction has been shown to induce malignant tumor phenotypes such as glycolytic phenotype, metastasis, drug resistance, and angiogenesis $(26,27)$. Ishikawa et al. showed that mtDNA mutations accelerate tumor metastatic activity, and resulted in ROS accumulation and induction of nuclear genes essential for tumor cell survival and angiogenesis (27). Mutations in mtDNA also induce tumor metastasis by promoting anti-apoptotic activities in tumor cells. In this case, mtDNA mutations constitutively activate the PI3K/Akt signaling pathway and protect tumor cells from stress-induced cell death (28). However, the causality between mtDNA mutations and drug resistance is poorly understood. In this study, we used the mtDNA-depleted cell line, $\rho^{0}$, to define the molecular mechanism of drug resistance induced by mitochondrial dysfunction. The $\rho^{0}$ cell line is a highly valuable model for studying diseases related to mitochondrial dysfunction (17). It has also been used to study drug resistance induced by mitochondrial dysfunction (29-31). Here, we found that mtDNA depletion decreases drug-induced cell death in HCT $116 / \rho^{0}$ cells (Fig. 1). Furthermore, the expression of pro-apoptotic proteins was reduced and the expression of anti-apoptotic proteins was increased. These results show that mtDNA depletion induces resistance to drug-induced cell death.

In recent years, several researchers reported that p53 targets mitochondria affecting both mtDNA abundance and mitochondrial homeostasis $(11,16)$. The p53 is known to maintain the genome function by acting as a transcription factor, DNA damage regulator, and signaling molecule (32). In addition to these roles, p53 acts as a mito-checkpoint protein and regulates mtDNA copy number and mitochondrial biogenesis (33). Furthermore, p53 plays a crucial role in the enhancement of base excision repair in mtDNA and localizes to the inner mitochondrial membrane fraction (34). Furthermore, mtDNA mutations and mitochondrial dysfunction are induced by mutated p53 in cancer cells. Interestingly, we observed that p53 expression was reduced in HCT116/ $\rho^{0}$ cells (Fig. 1 and 2). During HCT116/ $\rho^{0}$ cell generation, mtDNA depletion occurred prior to the reduction of p53 expression (Fig. 2) suggesting that mtDNA depletion induced reduction of $\mathrm{p} 53$ expression. Reduction of p53 expression by mtDNA depletion might accelerate oncogene and mtDNA mutations, and result in a drug-resistant phenotype in HCT $116 / \rho^{0}$ cells. To elucidate the molecular mechanism of p53 reduction in HCT116/ $\rho^{0}$ cells, we examined the intracellular levels of $\mathrm{NADH}$ and calcium. As $\mathrm{NADH}$ is an essential substrate for the mitochondrial electron transport chain, mtDNA mutations in the electron transport chain genes lead to NADH accumulation in the cytosol. Mitochondrial calcium is also released into the cytosol from collapsed mitochondrial structures in HCT116/ $\rho^{0}$ cells. Although both intracellular $\mathrm{NADH}$ and calcium levels were elevated in $\rho^{0}$ cells, the reduction of p53 expression was affected by elevated cytosolic calcium and not NADH levels (Fig. 3). Furthermore, NF-кB activation by elevated calcium levels in $\mathrm{HCT} 116 / \rho^{0}$ cells induced the reduction of p53 expression (Fig. 4). On the other hand, elevated NADH level in glycolytic $\rho^{0}$ cells may also be protective against drug-induced cell death. Pelicano et al. reported that $\rho^{0}$ cells show dependency on glycolysis, increased NADH level, and activation of the Akt kinase, leading to drug resistance and survival in case of hypoxic stress (22). They suggested that elevated NADH level in $\rho^{0}$ cells activates Akt via inactivation of the PTEN protein through a redox modification. We also observed the activation of Akt in HCT116/ $\rho^{0}$ cells (data now shown). Elevated NADH levels may activate Akt activity via PTEN inactivation in HCT116/ $\rho^{0}$ cells. Taken together, we hypothesize that resistance to drug-induced cell death in HCT116/ $\rho^{0}$ cells results from both reduction in p53 expression and activation of Akt as shown in Fig. 4D.

In conclusion, we demonstrated that mitochondrial dysfunction induced by mtDNA depletion causes drug resistance via a reduction in p53 expression. We found that elevated intracellular calcium levels significantly down-regulated p53 expression via NF- $\kappa B$ activation in the mtDNA-depleted HCT116/ $\rho^{0}$ cell model. Consequently, these data provide a novel mechanism for drug resistance triggered by mitochondrial dysfunction, and serve as a foundation for further studies and for the development of new cancer treatment strategies targeting the mitochondria.

\section{MATERIALS AND METHODS}

\section{Materials}

Dulbecco's Modified Eagle's medium, fetal bovine serum, antibiotics (penicillin and streptomycin), 10X Trypsin/ethylenediaminetetraacetic acid solution, and phosphate-buffered saline (PBS) were purchased from JBI (Daegu, Korea). All oligonucleotide primers for reverse transcription-polymerase chain reaction (RT-PCR) were generated by Cosmogenetech (Seoul, Korea). Antibodies were purchased from the following suppliers: Cell Signaling Technology, Danvers, MA, USA

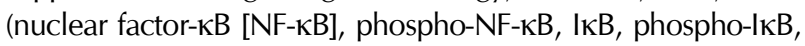
and p21) and Santa Cruz Biotechnology, Dallas, Texas, USA (p53, $\alpha$-tubulin, and secondary antibodies for rabbit and mouse). Fluo-3/AM was purchased from Invitrogen (Carlsbad, CA, USA). 


\section{Cell culture and generation of the HCT116/ $\rho^{0}$ cell line}

The human colon cancer cell line, HCT116, was obtained from American Type Culture Collection (Manassas, VA, USA) and maintained in RPMI1640 medium supplemented with $10 \%$ heat-inactivated fetal bovine serum and $1 \%$ penicillin/streptomycin at $37^{\circ} \mathrm{C}$ with $5 \% \mathrm{CO}_{2} . \mathrm{HCT} 116 / \rho^{0}$ cell line was generated as previously described (17). Briefly, HCT116 cells were incubated for approximately 50 days with media including $1 \mu \mathrm{g} / \mathrm{ml}$ ethidium bromide (EtBr), $50 \mu \mathrm{g} / \mathrm{ml}$ sodium uridine, and $1 \mathrm{mM}$ sodium pyruvate. Subsequently, the HCT116/ $\rho^{0}$ cell line was selected by treatment with rotenone and antimycin A (Sigma-Aldrich, St. Louis, MO, USA). Mitochondrial DNA depletion was analyzed by RT-PCR. After selection of the HCT116/ $\rho^{0}$ cell line, the cells were incubated in EtBr-free medium.

\section{Reverse transcription-polymerase chain reaction}

Total RNA was isolated from cells using TRIzol reagent (Invitrogen, Carlsbad, CA, USA) and converted to CDNA with M-MLV reverse transcriptase (Promega, San Luis, CA, USA) according to the manufacturer's instructions. Equal amounts of cDNA were subsequently amplified by PCR using the following primers: ND 1: 5'-GAG CAG TAG CCC AAA CAA-3' and 5'-TAG GGT GAG TGG TAG GAA GT-3'; ND 2: 5'-ACC CGT CAT CTA CTC TAC CA-3' and 5'-TAA TCC ACC TCA ACT GCC-3'; COX 1: 5'-TTG AAC AGT CTA CCC TCC C-3' and 5'-GCT CAC ACG ATA AAC CCT-3'; COX 2: 5'- CGT CTG AAC TAT CCT GCC-3' and $5^{\prime}$-GTC GTG TAG CGG TGA AAG-3'; COX 3: 5'-GAA AGC ACA TAC CAA GGC-3' and 5'-GCG GAT GAA GCA GAT AGT-3'; ATP 6: 5'-GTT CGC TTC ATT CAT TGC- $3^{\prime}$ and 5'-TGA GTA GGC TGA TGG TTT C-3'; CYTB: 5'- CAC TCC ACC TCC TAT TCT TG-3' and 5'-CTT ACT GGT TGT CCT CCG AT-3'; p53: 5'-TTT GCG TGT GGA TTT GG-3' and 5'-TTT TTA TGG CGG GAG GTA GA-3'; $\beta$-actin: 5'-GTG GGG CGC CCC AGG CAC CAG GGC-3' and 5'-CTC CTT AAT GTC ACG CAC GAT TTC-3'.

\section{Western blotting analysis}

Cells were lysed using radioimmunoprecipitation assay buffer containing $1 \mathrm{mM}$ phenylmethylsulfonyl fluoride, $1 \mathrm{mM}$ dithiothreitol, and protease and phosphatase inhibitor cocktails on ice for 20 mins. The protein samples were separated on $8-10 \%$ sodium dodecyl sulfate-polyacrylamide gels and transferred onto polyvinylidene fluoride membrane using a Trans-Blot semi-dry transfer kit (Bio-Rad, Hercules, CA, USA). The membranes were incubated for $2 \mathrm{~h}$ with the following primary antibodies: anti-mouse p53 (1:2,000), anti-mouse $\alpha$-tubulin $(1: 5,000)$, anti-rabbit NF-KB (1:500), and anti-rabbit phospho-NF-KB (1:500). Next, the membranes were washed three times with TBS $+0.1 \%$ Tween-20 for 10 mins each, after which they were incubated with horseradish peroxidaseconjugated anti-mouse or anti-rabbit antibody $(1: 5,000)$ for 30 mins. The membranes were then washed 4 times with TBS + $0.1 \%$ Tween-20 for 10 mins each. Immunoreactive bands were visualized using the ECL system.

\section{Measurement of intracellular NADH and calcium}

Cells $\left(40,000\right.$ cells $\left./ \mathrm{cm}^{2}\right)$ were seeded on $60-\mathrm{mm}$ dishes. After $24 \mathrm{~h}$, the cells were harvested using $1 \mathrm{ml}$ of PBS. Lysates were added to 3 wells in a 96-well plate. Measurements were conducted in triplicate for 3 individual experiments. The intracellular NADH level was measured by quantifying the intrinsic fluorescence under ultraviolet excitation using a spectrophotometer equipped with $340 \mathrm{~nm}$ excitation and 460 $\mathrm{nm}$ emission. The intracellular calcium level was measured by loading Fluo-3/AM, using a fluorometer at an excitation wavelength of $488 \mathrm{~nm}$ and an emission wavelength of 520 $\mathrm{nm}$.

\section{MTT assay}

Cells were seeded at a density of $4 \times 10^{4}$ cells/well in a 24-well plate. Seeded cells were incubated for $24 \mathrm{~h}$ in an incubator containing a humidified atmosphere of $5 \% \mathrm{CO}_{2}$. The viability assay was conducted using doxorubicin $(1 \mu \mathrm{M})$ or taxol $(10 \mathrm{nM})$ as a treatment followed by incubation at $37^{\circ} \mathrm{C}$ for $1 \mathrm{~d}$ and $3 \mathrm{~d}$. A final concentration of $0.6 \mathrm{mg} / \mathrm{ml}$ of MTT reagent was added directly to each well. After $4 \mathrm{~h}$, the medium was removed, the formazan crystals in cells were dissolved in dimethyl sulfoxide (DMSO), and the absorbance of the formazan solution was measured using an ELISA reader (Molecular Devices, Sunnyvale, CA) equipped with a $540 \mathrm{~nm}$ filter. Each sample was assayed in triplicate, and the experiment was repeated three times.

\section{Statistical analysis}

Data are presented as the means \pm standard deviation (SD). Statistical comparisons between groups were performed using Student's t-test. $\mathrm{P}<0.05$ was considered statistically significant. Statistical analyses were performed in Microsoft Excel.

\section{ACKNOWLEDGEMENTS}

This research was supported by Basic Science Research Program through the National Research Foundation of Korea (NRF) funded by the Ministry of Education, Science and Technology (NRF-2016R1D1A1B01015151).

\section{CONFLICTS OF INTEREST}

The authors have no conflicting interests.

\section{REFERENCES}

1. Scatena R (2012) Mitochondria and cancer: a growing role in apoptosis, cancer cell metabolism and dedifferentiation. Adv Exp Med Biol 942, 287-308

2. Um JH and Yun J (2017) Emerging role of mitophagy in human diseases and physiology. BMB Rep 50, 299-307 
3. Cho B and Sun W (2017) CoMIC, the hidden dynamics of mitochondrial inner compartments. BMB Rep 50, 597-598

4. Taanman JW (1999) The mitochondrial genome: structure, transcription, translation and replication. Biochim Biophys Acta 1410, 103-123

5. Brandon M, Baldi P and Wallace DC (2006) Mitochondrial mutations in cancer. Oncogene 25, 4647-4662

6. Chatterjee A, Mambo E and Sidransky D (2006) Mitochondrial DNA mutations in human cancer. Oncogene 25, 4663-4674

7. Chen El (2012) Mitochondrial dysfunction and cancer metastasis. J Bioenerg Biomembr 44, 619-622

8. Boland ML, Chourasia AH and Macleod KF (2013) Mitochondrial dysfunction in cancer. Front Oncol 3, 292

9. Kim N, Min WK, Park MH, Lee JK, Jin HK and Bae JS (2016) Neuropeptide $Y$ protects kidney against cisplatininduced nephrotoxicity by regulating p53-dependent apoptosis pathway. BMB Rep 49, 288-292

10. Brosh R and Rotter V (2009) When mutants gain new powers: news from the mutant p53 field. Nat Rev 9, 701-713

11. Koczor CA, White RC, Zhao P et al (2012) p53 and mitochondrial DNA: their role in mitochondrial homeostasis and toxicity of antiretrovirals. Am J Pathol $180,2276-2283$

12. Bourdon A, Minai L, Serre V et al (2007) Mutation of RRM2B, encoding p53-controlled ribonucleotide reductase (p53R2), causes severe mitochondrial DNA depletion. Nat Genet 39, 776-780

13. Charlot JF, Pretet JL, Haughey C and Mougin C (2004) Mitochondrial translocation of p53 and mitochondrial membrane potential (Delta Psi m) dissipation are early events in staurosporine-induced apoptosis of wild type and mutated p53 epithelial cells. Apoptosis 9, 333-343

14. Wang W, Sun Q, Wu Z et al (2013) Mitochondrial dysfunction-related genes in hepatocellular carcinoma. Front Biosci (Landmark edition) 18, 1141-1149

15. Bode AM and Dong Z (2004) Post-translational modification of p53 in tumorigenesis. Nat Rev 4, 793-805

16. Galluzzi L, Morselli E, Kepp O et al (2008) Targeting p53 to mitochondria for cancer therapy. Cell Cycle 7, 1949-1955

17. Miceli MV and Jazwinski SM (2005) Common and cell type-specific responses of human cells to mitochondrial dysfunction. Exp Cell Res 302, 270-280

18. Mansfield KD, Guzy RD, Pan Y et al (2005) Mitochondrial dysfunction resulting from loss of cytochrome $\mathrm{c}$ impairs cellular oxygen sensing and hypoxic HIF-alpha activation. Cell Metab 1, 393-399

19. Kemp CJ, Donehower LA, Bradley A and Balmain A (1993) Reduction of p53 gene dosage does not increase initiation of promotion but enhances malignant progression of chemically induced skin tumors. Cell 74,
813-822

20. Zhu Y, Guignard F, Zhao D et al (2005) Early inactivation of p53 tumor suppressor gene cooperating with NF1 loss induces malignant astrocytoma. Cancer Cell 8, 119-130

21. Butow RA and Avadhani NG (2004) Mitochondrial signaling: the retrograde response. Mol Cell 14, 1-15

22. Pelicano $H, X u R H, D u M$ et al (2006) Mitochondrial respiration defects in cancer cells cause activation of Akt survival pathway through a redox-mediated mechanism. J Cell Biol 175, 913-923

23. Tergaonkar V, Pando $M$, Vafa $O$ et al (2002) p53 stabilization is decreased upon NFkappaB activation: a role for NFkappaB in acquisition of resistance to chemotherapy. Cancer Cell 1, 493-503

24. Huang WC, Ju TK, Hung MC and Chen CC (2007) Phosphorylation of CBP by IKKalpha promotes cell growth by switching the binding preference of CBP from p53 to NF-kappaB. Mol cell 26, 75-87

25. Ferran C, Millan MT, Csizmadia $V$ et al (1995) Inhibition of NF-kappa B by pyrrolidine dithiocarbamate blocks endothelial cell activation. Biochem Biophys Res Commun 241, 212-223

26. Wallace DC (2012) Mitochondria and cancer. Nature Rev $12,685-698$

27. Ishikawa K, Takenaga K, Akimoto M et al (2008) ROS-generating mitochondrial DNA mutations can regulate tumor cell metastasis. Science NY 320, 661-664

28. Kulawiec M, Owens KM and Singh KK (2009) Cancer cell mitochondria confer apoptosis resistance and promote metastasis. Cancer Biol Ther 8, 1378-1385

29. Vaux EC, Metzen E, Yeates KM and Ratcliffe PJ (2001) Regulation of hypoxia-inducible factor is preserved in the absence of a functioning mitochondrial respiratory chain. Blood 98, 296-302

30. Naito A, Cook CC, Mizumachi T et al (2008) Progressive tumor features accompany epithelial-mesenchymal transition induced in mitochondrial DNA-depleted cells. Cancer Sci 99, 1584-1588

31. Ishikawa F, Kaneko E, Sugimoto $T$ et al (2014) A mitochondrial thioredoxin-sensitive mechanism regulates TGF-beta-mediated gene expression associated with epithelial-mesenchymal transition. Biochem Biophys Res Commun 443, 821-827

32. Efeyan A and Serrano $M$ (2007) p53: guardian of the genome and policeman of the oncogenes. Cell Cycle 6 , 1006-1010

33. Kulawiec M, Ayyasamy $V$ and Singh KK (2009) p53 regulates mtDNA copy number and mitocheckpoint pathway. J Carcinogenesis 8, 8

34. Chen D, Yu Z, Zhu Z and Lopez CD (2006) The p53 pathway promotes efficient mitochondrial DNA base excision repair in colorectal cancer cells. Cancer Res 66, 3485-3494 